\title{
Flux transport dynamo coupled with a fast tachocline scenario
}

\author{
Bidya Binay Karak ${ }^{1}$ and Kristof Petrovay ${ }^{2}$ \\ ${ }^{1}$ Department of Physics, Indian Institute of Science, Bangalore 560012, India \\ email: bidya_karak@physics.iisc.ernet.in \\ ${ }^{2}$ Eötvös University, Department of Astronomy, Budapest, Hungary
}

\begin{abstract}
The tachocline is important in the solar dynamo for the generation and the storage of the magnetic fields. A most plausible explanation for the confinement of the tachocline is given by the fast tachocline model in which the tachocline is confined by the anisotropic momentum transfer by the Maxwell stress of the dynamo generated magnetic fields. We employ a flux transport dynamo model coupled with the simple feedback formula of this fast tachocline model which basically relates the thickness of the tachocline to the Maxwell stress. We find that this nonlinear coupling not only produces a stable solar-like dynamo solution but also a significant latitudinal variation in the tachocline thickness which is in agreement with the observations.
\end{abstract}

Keywords. Sun: dynamo, Sun: tachocline, Sun: magnetic fields.

\section{Introduction}

The tachocline is a thin layer located at the base of the solar convection zone where the rotation changes from differential to the rigid rotation. However the thinness of this layer made the confinement of the tachocline an intriguing problem. One plausible explanation for the confinement of the tachocline was that the Maxwell stress of the dynamo generated magnetic fields can provide a strong anisotropic angular momentum transport in the horizontal direction. This is known as the fast tachocline mechanism (Forgacs-Dajka \& Petrovay 2001, 2002; Forgacs-Dajka 2003).

In the fast tachocline scenario, the thickness of the tachocline depends on the magnetic field in a nonlinear way. On the other hand, the thickness of the tachocline is an important input parameter of flux transport dynamo models which is successful in explaining many important aspects of the solar cycle (Chatterjee, Nandy \& Choudhuri 2004; Choudhuri \& Karak 2009; Karak 2011; Karak \& Choudhuri 2011, 2012; Choudhuri \& Karak 2012; Karak \& Nandy 2012). The objective of the present work is to couple the simple feedback formula capturing the essential physics of the fast tachocline model in a flux transport dynamo model and to see its response in flux transport dynamo model. Details can be found in Karak \& Petrovay (2012).

\section{Results}

Following Forgács-Dajka \& Petrovay (2001) the approximate relation between the mean (cycle averaged) tachocline thickness and Maxwell stress can be written as

$$
d_{t}^{2}=\frac{C \eta_{t}}{\bar{B}_{p}(\theta, t) \bar{B}(\theta, t)} .
$$

where $C \approx 2 \times 10^{15} \mathrm{G}^{2} \cdot \mathrm{s}, \eta_{t}$ is the mean diffusivity in the tachocline and $\bar{B}$ and $\bar{B}_{p}$ $\left(=\sqrt{B_{r}^{2}+B_{\theta}^{2}}\right)$ are the local radially averaged values of the toroidal and the poloidal 

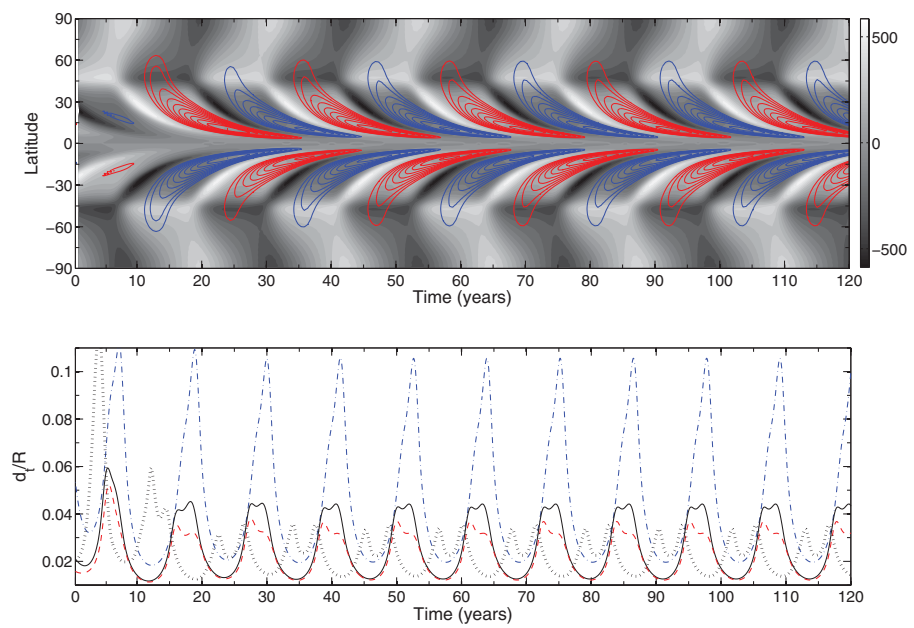

Figure 1. Top: butterfly diagram of magnetic fields for variable $d_{t}$ as given by Eq. (2.1). Contours show the butterfly diagram of the toroidal field in the tachocline. The background shows the radial field on the solar surface. Bottom: variation of $d_{t}$ with time. The dash-dotted, solid, dashed and dotted lines are the values at $75^{\circ}, 60^{\circ}, 45^{\circ}$, and $15^{0}$ latitudes, respectively.

field. We use this relation for the tachocline thickness in a flux transport dynamo model. For the dynamo calculations we use the Surya code (Chatterjee, Nandy \& Choudhuri 2004) with modified parameters presented in Karak \& Petrovay (2012). The result is shown in Figure 1. It is interesting to note is that this produces a stable solar-like dynamo solution. In addition, it produces a significant variation in the tachocline thcikness with latitude and time. The thickness of the tachocline varies from $0.02 R$ to $0.1 R$ as we move from low to high latitudes which is in agreement with the observations (e.g., Antia \& Basu 2011). However the solar cycle variation of tachocline thickness is quite significant, and somewhat higher than what the observational constraints suggest.

\section{Acknowledgements}

This work was supported by the Hungarian Science Research Fund (OTKA grants no. K83133 and K81421). BBK thanks Department of Science and Technology, Government of India for providing the travel support to participate this symposium.

\section{References}

Antia, H. M. \& Basu, S.: 2011, ApJ Lett. 735, L45

Chatterjee, P., Nandy, D., \& Choudhuri, A. R. 2004, A\&A A, 427, 1019

Choudhuri, A. R. \& Karak, B. B. 2009, RAA, 9, 953

Choudhuri, A. R. \& Karak, B. B. 2012, Phys. Rev. Lett., 109, 171103

Forgács-Dajka, E. \& Petrovay, K. 2001, Solar Phys. 203, 195

Forgács-Dajka, E. \& Petrovay, K. 2002, A\&A 389, 629

Forgács-Dajka, E. 2003, A\&\&A 413, 1143

Karak, B. B. 2010, ApJ, 724, 1021

Karak, B. B. \& Choudhuri, A. R. 2011, MNRAS, 410, 1503

Karak, B. B. \& Choudhuri, A. R. 2012, Solar Phys., 278, 137

Karak, B. B. \& Petrovay, K. 2013, Solar Phys., 282, 321

Karak, B. B. \& Nandy, D. 2012, ApJ Lett., 761, L13 